\title{
Factores inmunológicos en la diarrea infantil*
}

\author{
Dls. Ernesto Guirakles*", Ricardo Sörensen"**, \\ Liliana Rebolledo****. Amanda Carmona***, \\ Carmen Gutierrez.**
}

El tracto digestivo, mirado en conjunto, constituye un importante componente del mecanismo de defensa inmunológico del organismo. (1-3) Así, una serie de enfermedades gastrointestinales han sido vinculadas a alteración de estos sistemas inmunológicos, (2-5) aunque creemos que en la mayoría de los casos esta interrelación está todavía en la etapa de las interrogantes.

La existencia de un sistema basado principalmente en inmunoglobulinas secretoras, hace de la submucosa intestinal una eficiente barrera protectora contra agentes infecciosos y también contra otros antígenos (alimentarios, por ejemplo). (1, 3, 6, 7) La inmunoglobulina A, secretada a lo largo del intestino, es elaborada por las células plasmáticas de la submutcosa y complementada con el componente secretorio que le da sus características particu-

*Trabajo financiado parcialmente por el Proyecto N? 1171 de la Oficina Técníca de Desarrollo Científico y Creación Artística de la Universidad de Chile. Presentado como Relato Oficial en las V Jornadas Anuales de la Sociedad Chilena de Pediatría, Lo Barnechea, 1975.

* Departamento de Pediatría, Universidad de Chile, Sede Oriente, Hospital Luis Calvo Mackenna.

"*Departamento de Inmunología Instituto Bacteriológico de Chile y Unidad de Inmunología Hosp. Luis Calvo Mackenna.

${ }^{* * * *}$ Q.F.. Departamento de Pediatría, Universidad de Chile, Sede Oriente. lares. $(1,3)$. Así, de dos monómeros de inmunoglobulina A unidos por el componente secretorio resulta un dímero, que es propiamente la molécula secretada. Esta inmunoglo bulina parece representar el factor individual más importante del aparato inmunológico intestinal. Por tal razón se comprenderá que la deficiencia selectiva de IgA, así como la deficiencia combinada de ésta y otras inmunoglobulinas se acompañan de alteraciones, a veces severas, de la función intestinal.

En las exposiciones siguientes se verá en más detalle algunos ejemplos de las inmunodeficiencias ya aiudidas. Es interesante acotar que en estas enfermedades es relativamente frecuente el hallazgo de infestaciones severas y recurrentes por Giardia Lambia. $(1,4,8)$ Aunque este protozoo no sea la única causa aparente de mala absorción intestinal con alteraciones histológicas de la mucosa, (8) no hay duda que es el agente individual de mayor importancia.

Pasando a otras condiciones que cursan con diarrea crónica, es interesante acotar que, con respecto a Enfermedad Celíaca, las investigaciones actuales apuntan hacia una patogénesis inmunológica, $(9,10)$ Se postula que el gluten desencadena una respuesta inmunológica local en el individuo susceptible, lo que lleva a infiltración celular del corión (principalmente por células plasmáticas), daño epitelial, y finalmente atrofia de las vellosidades. Hay varias otras evidencias en favor de un mecanismo inmunológico en la enteropatía por 
gluten. Por ejemplo, la asociación de Enfermedad Celíaca y deficiencia selectiva de IgA es mucho más frecuente de lo qưe cabría esperar por simple coincidencia. (4) Por otra parte, según estudios recientes, el antígeno de histocompatibilidad HL-A8 aparece en proporción varias veces superior en los pacientes celíacos que en el resto de la población. $(11,14)$

Con respecto a otros cuadros gastrointestinales con presunta etiopatogenia inmunologica, $(3,5)$ cabe destacar que en este grupo actualmente se incluye a la Enfermedad de Crohn, la colitis ulcerosa, la anemia perniciosa con gastritis atrófica, además de otras entidades mucho menos comunes.

En varios casos publicados en la literatura en los últimos años, $(15,17)$ se ha descrito la asociación de fallas de opsonización, con diarrea infantil de curso grave e "intratable", infecciones a repetición y moniliasis mucocutánea. En el clásico trabajo de Leiner, (18) publicado hace ya más de 60 años, se puede encontrar descripciones que calzarían con éstas más recientes.

En el Hospital Luis Calvo Mackenna hemos estudiado recientemente desde un punto de vista inmunológico y microbiológico varios casos de diarrea prolongada del lactante. En estos casos se incluyen algunos que cumplen con las características clínicas recién enunciadas.

El objetivo de este trabajo es dar a conocer los resultados preliminares obtenidos en este estudio.

Inicialmente fueron estudiados 2 casos en los cuales la presentación de la enfermedad tenia hechos comunes que son sintetizados en la Tabla No 1 (casos 1 y 2). En ambos lactantes la diarrea se inició a los pocos días del destete, siguiendo un curso artastrado y llevándolos a la desnutrición progresiva a pesar de las diferentes medidas díetéticas y quimioterapeuticas empleadas. En ambos se present6 la moniliasis mucocutánea progresiva casi desde el principio de la diarrea, y durante la evolución de ésta se futeron agregando infecciones en distintos sitios. En el paciente $N: 1$ existio intolerancia a hidratos de carbono, que comprometió incluso la absorción de monosa- cáridos, en tanto que en el caso 2 la alteración se restringió a los disacáridos. Los hallazgos de laboratorio más relevantes aparecen descritos en la T'abla $N$ : 2.

Puede observarse que el hecho más resaltante del estado inmunológico de ambos pacientes consiste en la simultánea alteración del complemento hemolítico y de la fagocitosis de levaduras. La terapia con plasma fresco en ambos casos contribuyó a la mejoría clínica, y además en el caso 1 produjo normalización in-vitro de la fagocitosis de levaduras; (el caso 2 no fue estudiado desde este punto de vista).

Los resultados del estudio inmunológico en estos dos pacientes motivaron la extensión de la investigación a 8 lactantes que también presentaron diarrea prolongada. El caso No 3 manifestó sus síntomas a partir del período de destete. En los otros casos, la iniciación de la diarrea no tuvo relación aparente con este hecho, y en algunos prácticamente no existio lactancia materna. La Tabla No 1 resume algunos hechos importantes de la historia clínica de estos 8 lactantes (casos 3 al 10). En todos ellos la diarrea siguió una evolución prolongada y refractaria a los tratamientos habituales.

Los estudios inmunológicos efectuados aparecen descritos en la Tabla № 2 . Se observa que en tres de estos 8 lactantes se detectó inmunodeficiencias similares a las encontradas en los casos 1 y 2 . En un cuarto paciente (caso № 10) las alteraciones inmunológicas consistieron en ausencia de los linfocitos $\mathbf{T}$. También en estos 8 lactantes hubo severo compromiso de la absorción intestinal de carbohidratos. Los pacientes N.os 3 y 4 presentaron intolerancia total a los hidratos de carbono (monosacáridos y disacáridos), en tanto que en el resto, el compromiso se circunscribio a los disacáridos.

\section{COMENTARIO}

Los hallazgos inmunologicos en estos 10 casos de síndrome diarreico de evolución prolongada nos permiten destacar lo siguiente:

a) Inmunoglobulinas: las cifras globales de 
IgM, JgG e IgA se mantuvieron siempre dentro de límites normales en todos los pacientes. Esto no excluye incapacidad de formar anticuerpos especificos, hecho que no fue investigado en forma dirigida.

b) Inmunidad celular: a pesar de la desnutrición secundaria de los 10 casos, en 3 hubo reacciones intradérmicas positivas, to que hace poco probable un rol preponderante de una deficiencia celular en la predisposición a la diarrea prolongada. En este mismo sentido se debe valorar la ausencia de diarrea en muchos desnutridos con franca alteración de la inmunidad celular. (19)

Merecen destacarse aquí: la alteración específica de la reacción a candidiasis en presencia de reacciones normales a dinitroclorobenceno en el caso № 1 ; la ausencia de linfocitos $T$ en un lactante (caso 10) con linfocitos circulantes normales. En él, aun en presencia de complemento hemolítico normal, hubo una incapacidad de ingerir partículas de candida, hecho que podría ser atribuido a una falta de activación de los fagocitos por parte de los linfocitos $\mathbf{T}$.

c) Complemento hemolítico y fagocitosis: a diferencia del caso recién mencionado, en 5 de los 10 pacientes se encontró una asociación entre incapacidad de ingerir partículas de levaduras y alteración del complemento. La determinación de la actividad hemolítica global del complemento no indica, en caso de alteración, cuál es el factor deficitario*. Dado el rol de C5 en la opsonización de partículas de levaduras, podría postularse que ése ha sido el factor alterado. $(15,17)$ En ninguno de los pacientes estudiados se encontró defectos metabolicos del fagocito por medio de la prueba del NBT (nitrobluetetrazolium).

La anormalidad descrita para los anteriores 5 pacientes es susceptible de corregirse in-vitro con la plasma fresco normal, ya que éste no pierde los factores de complemento por inactivación. Los pacientes que fueron tratados de esta manera parecen haberse beneficiado

\footnotetext{
- $\mathrm{C}_{3}$ se encontró dentro de límites normales en
} esios pacientes. clínicamente. Los casos descritos parecen tener hechos en común con aqueilos publicados recientemente en la literatura, $(15,17)$ y a los cuales hacíamos alusión al principio.

El seguimiento de nuestros pacientes mostró que la alteración de la fagocitosis y del complemento fue transitoria, observándose una corrección de estos valores junto con la mejoría clínica.

El niño desnutrido, especialmente aquel que vive en ambientes contaminados, está sujeto al constante insulto de diversís agentes patógenos, tanto enterales como de otros sistemas. A esto se añade el rol deletéreo que adopta cierta flora intestinal (que en condiciones habituales es saprófita) cuando existe en cantidades excesivas, llevando a manifiestas alteraciones de las funciones digestivas. $(20,24)$ Estos mecanismos estân ciertamente involucrados en la génesis de diarreas infantiles de evolución prolongada y complicada. Especial relevancia tienen en este contexto la flora de tipo fecal y la candida albicans, microorganismos particularmente abundantes en el tracto intestinal de niños desnutridos que viven en condiciones de insalubridad. $(20,24)$ Las alteraciones inmunológicas, tanto primarias como secundarias favorecen asi la agresión permanente de microorganismos con potencialidad patógena en la iniciación y prolongación de la diarrea. En la patogenia multifactorial de estos curadros diarreicos de evolución prolongada, un factor casi siempre presente es la intolerancia a hidratos de carbono, $(25,26)$ condición que en sus grados más intensos (como lo es el compromiso de la absorción de monosacáridos) ensombrece notablemente el pronóstico de la enfermedad. (26, 28)

En resumen, en 5 de 10 casos de diarrea prolongada del lactante el defecto inmunologico más destacado reside en una alteración reversible del complemento hemolitico, que condiciona una menor capacidad de ingerir partículas de levadura. Esta entidad nosol6gica debe considerarse junto a las ya conocidas en las que hay deficiencias de IgA o alteraciones de inmunidad celular, predisponiendo así hacia una mayor susceptibilidad a las dia- 
rreas graves y a infecciones de diversos sistemas.

\section{SUMMARY}

The findings of an immunologic analisis of 10 children with protracted diarrhea are shown. Most notably, in 5 out of 10 infants aged 1 to 5 months, a severe impairment of the henolytic activity of complement was found. In every case, this was accompanied by a low phagocytic index for yeast particles. This alteration could be reserved in vitro with the addition of fresh normal plasma. In vivo, the clinical condition of some of these children was also improved by treatment with fresh plasma.

The different immunological alterations which could lead to chronic or protracted diarrhea in childhood are discussed.

Tabla Na 1

CARACTERISTICAS CLINICAS DE 10 LACTANTES CON DIARREA PROLONGADA

\begin{tabular}{|c|c|c|c|}
\hline Paciente & $N^{\circ}$ & Diagnósticos & Evolucion subsecuente \\
\hline L.G. & 1 & $\begin{array}{l}\text { Diarrea postdestete. Moniliasis. } \\
\text { Infeciones recurr. }\end{array}$ & $\begin{array}{l}\text { Favorable: linfocitos maternos } \\
\text { y plasma fresco. }\end{array}$ \\
\hline F.S. & 2 & $\begin{array}{l}\text { Diarrea postdestete. Dermati- } \\
\text { tis. Infecciones recurr. }\end{array}$ & Favorable con plasma fresco. \\
\hline J.L. & 3 & $\begin{array}{l}\text { Diarrea postdestete. Desnutri- } \\
\text { ción progresiYa. }\end{array}$ & Fallece. \\
\hline $\mathbf{L}, \mathbf{R}$. & 4 & $\begin{array}{l}\text { Diarrea prolongada. } \\
\text { Mucopolisacáridosis } \\
\text { Prematurez. Amaurosis. }\end{array}$ & Favorable. \\
\hline M.I. & 5 & $\begin{array}{l}\text { Diarrea prolongada } \\
\text { Bronconeumonia. }\end{array}$ & Favorable. \\
\hline S.S. & 6 & Diarrea prolongeda. & Favorable. \\
\hline J.L. & 7 & $\begin{array}{l}\text { Diatrea prolongada } \\
\text { Recrudescencia por } \\
\text { Salmonellosis. }\end{array}$ & Fallece. \\
\hline D.O. & $\mathbf{B}$ & Diarrea prolongada. & Fayorable. \\
\hline J.C. & 9 & Diarrea prolongada. & Favorable. \\
\hline H.S. & 10 & $\begin{array}{l}\text { Diarrea prolongada. } \\
\text { Reingresa por } \\
\text { Bronconeumonis. }\end{array}$ & $\begin{array}{l}\text { Fallece por } \\
\text { Bronconeumonia. }\end{array}$ \\
\hline
\end{tabular}


Tabla N: 2

HALLAZGOS INMUNOLOGICOS EN LACTANTES CON DIARREA PROLONGADA Y DESNUTRICION

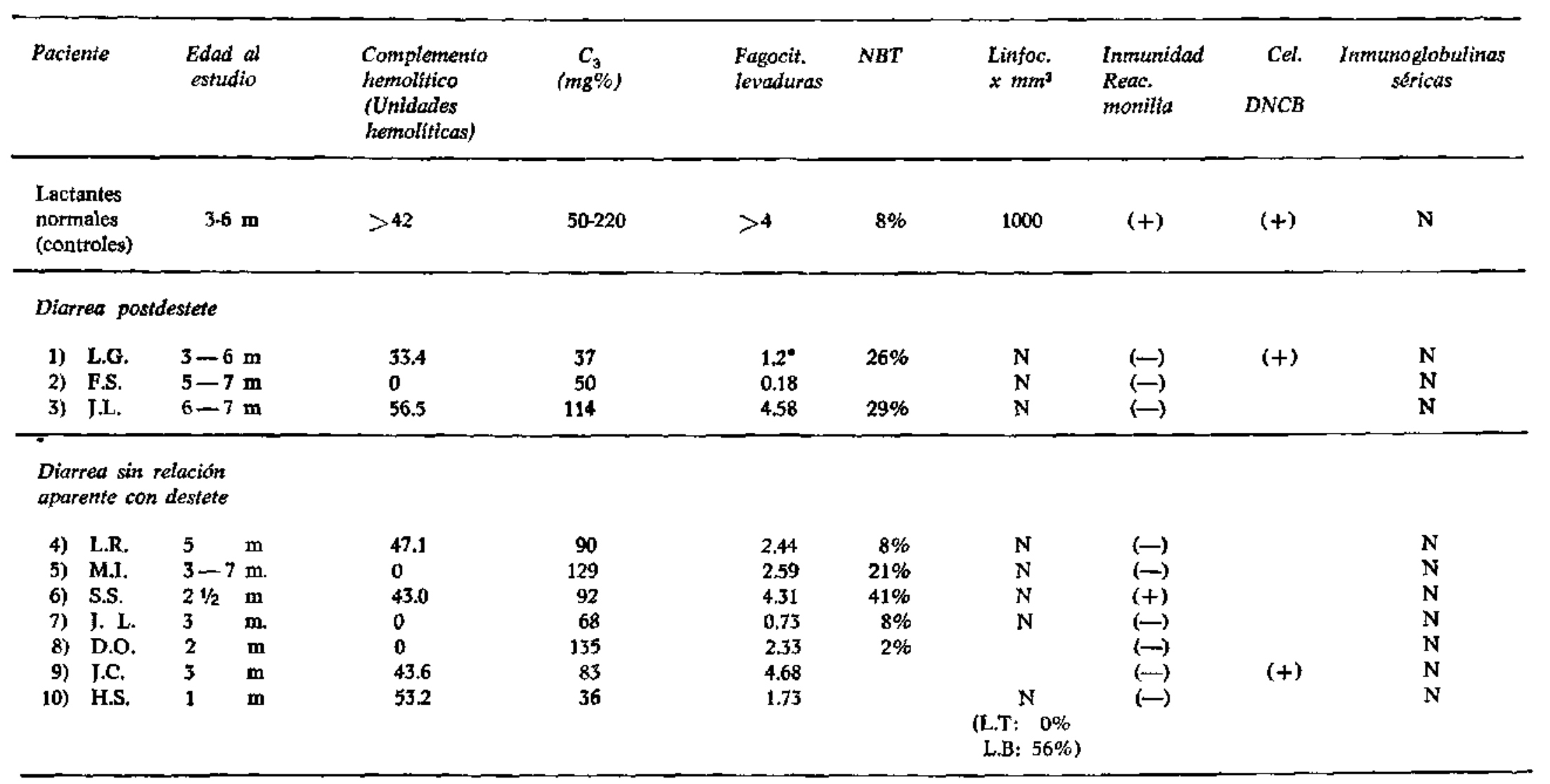

* La fagocitosis de particulas de levadura se corrigió al agregar plasma fresco de un individuo nor mal. 


\section{REFERENCIAS}

1. Walker, W.A. y Hong, R.: I. Pediatrics. 83: 517, 1973.

2. The Lymphocytc and Gut. Editorial. Lancet. 2: 31, 1974.

3. Silverman, A., Roy, C.C. y Cozzeto, F.J.: Inmune homeostasis and the gut. En: Pediatric Clinical Gastroenterology. C.V. Mosby Co., 1971.

4. Walker, W.A. y Hong, R.: T. Pediatrics. 83: $711,1973$.

5. Brandizsaeg, P. y cols.: Acta Pathol \& Microb. Scand., Supl, 248: 43, 1974.

6. Fubara, E.S. y Freter, R.: Amer. J. Clin. Nutr. 25: 1357, 1972.

7. Walker, W.A., Isselbacher, K.J. y Bloch, K.f.: Amer. J. Clin. Nutr. 27: 1434, 1974.

8. Ament, M.E. y Rubin, C.E.: Gastroenterology. 62: 216, 1972.

9. Anderson, C.M., Gracey, M. y Burke, V.: Arch. Dis. Childh, 47: 292, 1972.

10. Falchuk, Z.M. y Strober, W.: Gut, 15: 947, 1974.

11. Falchuk, Z.M., Rogentine, G.N. y Strober, W.: J. Clin. Invest. 5I: 1602, 1972.

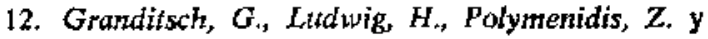
Wick, G.: Lancet. 2: 908, 1973.

13. Price-Evans, D.A.: Lancet. 2: 1096, 1973.

14. Scott, B.B. y cols.: Lancet. 2: 374, 1974.

15. Miller, M.E. y Nilsson, U.R.: New Engl. J. Med. 282: 354, 1970.
16. Jacobs, J.C. y Miller, M.E.: Pediatrics. 49: 225, 1972.

17. Scott, H., Moynahan, E.J., Risdon, R.A., Har yey, B.A.M. y Soothill, I.F.: Arch. Dis, Childh. 50: 311, 1975.

18. Leiner, C.: Archiy. fur Dermatologie und Syphjlis. 89: 163, 1908.

19. Chandra, R.T.: I. Pediatrics. 81: 1194, 1972.

20. Mafa, L.J., Urrutia, J.J. y Lechitig, A.: Amer. J. Clin. Nutr. 24: 249, 1971.

21. Mata, L.J., Jimenez, F., Cordon. M., Kosales, P., Prera, E., Schneider, R.E. y Viteri, F.: Amer. J. Clin. Nutr. 25: 1118, 1972.

22. Gracey, M. y Stone, D.E.: Aust. N.Z. J. Med. 3: 215, 1972.

23. Gracey, M., Suhariono, Sunoto y Slone, D.E.: Amer. J. Clin. Nutr. 26: 1170, 1973.

24. Gracey, M., Stone, D.E., Suharjono y Sunoto: Amer. J. Clin. Nutr. 27: 345, 1974.

25. Bowie, M.D., Brinkman, G.L. y Hansen, /.D.L.: J. Pediatrics 66: 1083, 1965.

26. Lifschitz, F, Coello-Ramirez, P., Gutiérrez-Topete, G. y Cornado-Comet, M.C.: Pediatrics. 79: 760,1971 .

27. Lifschitz, F., Coello-Ramírez, P. y Gutiérrez* Topete, G.: I. Pediatrics. 77: 595, 1970.

28. Macho, L., Guiraldes, E., Sörensen, R., Gutierrez, C., Lobas, $H$. y Harún, A.: Intolerancia a monosacáridos en el curso del síndrome diarreico agudo de evolución prolongada. En pre. paración, 1975. 